\title{
Implementation of incremental yield conditions in spherical coordinates
}

\author{
Sergey Kalashnikov*, Elena Gurova \\ Volgograd State Technical University, 400005, Volgograd, Russia
}

\begin{abstract}
The application of incremental plasticity conditions in the axisymmetric problem of a spherical tank loaded with internal and external pressure is considered. Inhomogeneous stress distribution leads to increased limiting gradient stresses, which depend on the internal cavity size. For the tanks of real dimensions, the magnification limits are established for different laws of approximation of the deformations' constraint gradient effect.
\end{abstract}

\section{Introduction}

Any theory of strength in the structures design is aimed at solving the problem of ensuring the trouble-free operation parameters and at the same time reducing material consumption. Ensuring safe operation at all stages of the life cycle is achieved by assessing the permissible limit state. For example, for the structures made of plastic materials, such a state is the onset of plastic deformations in a dangerous section, where the greatest voltage values are achieved. In this case, the calculated stress state of the structure is compared with the calculated resistance of the material. The limiting stage of deformation is normalized by the achievement of the maximum stress of a value lower than the design resistance of the material, which is essentially a practical implementation of the plasticity condition. Such computational models are based on the physical laws of deformation obtained by testing samples for uniaxial tension. In the field of elastic deformations, this model satisfies the criterion of practice and gives the simplest linear mathematical approximation. However, the experimental data analysis [1-5] shows that the yield stress of the material determined from similar experiments does not allow to reliably solve the problems of strength with an inhomogeneous stress distribution, when there is constraint of shear deformations along sliding areas in the areas with the stress gradients, which leads to the effect increasing the limiting elastic stresses.

\section{Main part}

In this work, the incremental plasticity conditions are applied for a homogeneous isotropic material with a yield area [6-9]. The form of Tresca or von Mises criteria is used in the conditions, but the yield onset is determined by the achievement of the maximum stress at the

\footnotetext{
* Corresponding author: kalashnikovsu@mail.ru
} 
dangerous point of a certain increased gradient value, a larger yield stress of the material. This increase will have the greater degree, the more inhomogeneous the stress distribution in the vicinity of the point under consideration is and for its approximation an asymptotic dependence is proposed:

$$
T_{g r}=T_{0}+\left(T_{m}-T_{0}\right) \frac{g}{\lambda_{T, g}+g} .
$$

It is written in shear stress intensities for the von Mises condition. In (1) $g=\operatorname{grad} T / T$ is a measure of stress state inhomogeneity; $T_{m}$ is the largest of the stresses possible in a nonuniform stress state, which is taken equal to $1.5 T_{0} ; \lambda_{T, g}$ is the elastic characteristic of a material with a dimension inverse to its length. In $[9,10]$, from the experimental data [2] for the bent steel beams, $\lambda_{T, g}=20.1587 \mathrm{~m}^{-1}$ was obtained.

The proposed gradient approach is implemented in solving the problems of the strength of rod elements in bending [9-12], eccentric compression [11]. A distinctive feature of this condition application is obtaining, as a solution, either a resolving equation or an algebraic expression that has an unambiguous interpretation: depending on the geometric parameters and the design scheme, the onset of plastic deformations in dangerous sections corresponds to the only possible value of the increased gradient stress.

Spherical tank with outer radius $b$ and inner radius $a$ are generally loaded with internal $p$ and external $q$ pressures (Fig. 1). We use a spherical coordinate system with the origin at the center [13]. The stress state in the walls of the vessel is inhomogeneous and under the action of internal pressure according to the well-known Lamé solution is determined by the expressions $[14,15]$ :

$$
\sigma_{r}=C \cdot \frac{b^{3}-r^{3}}{r^{3}} ; \sigma_{\varphi}=\sigma_{\theta}=-C \cdot \frac{2 r^{3}+b^{3}}{2 r^{3}}
$$

where

$$
C=\frac{p a^{3}}{a^{3}-b^{3}}
$$

Due to symmetry $\sigma_{\varphi}=\sigma_{\theta}, \tau_{r \varphi}=\tau_{r \theta}=\tau_{\varphi \theta}=0$ and the intensity of shear stresses for any point:

$$
T=\frac{1}{\sqrt{3}} \sqrt{\left(\sigma_{r}-\sigma_{\varphi}\right)^{2}}
$$

or, taking into consideration (2),

$$
T=\sqrt{3 C^{2}} b^{3} / 2 r^{3}
$$

in the case under consideration 


$$
\operatorname{grad} T=\left|\frac{\partial T}{\partial r}\right|=\frac{3 \sqrt{3 C^{2}} b^{3}}{2 r^{4}},
$$

which determines the function of the stress state inhomogeneity standard

$$
\frac{\operatorname{grad} T}{T}=3 / r
$$

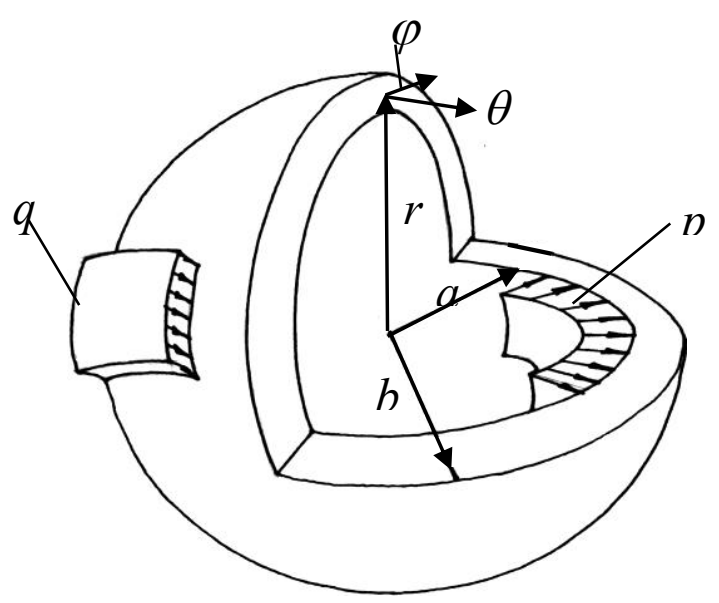

Fig. 1. Design diagram of a spherical tank

Substituting this result into the dependence (1) for the plasticity condition and, taking into account that the first plastic deformations appear on the inner surface of the reservoir at $r=a$, where value $T$ is as big as possible, we get the following analytical dependence:

$$
T_{g r}=T_{0}\left(1+\frac{3}{2 \lambda_{T, g} a+6}\right)
$$

which can be interpreted as follows: each tank with the radius of the inner cavity $a$ will correspond to a certain value of the intensity of shear stresses $T_{g r}$, at which plastic deformations begin. The value of the corresponding internal pressure is determined by equating the right-hand sides (3) and (4). Denoting relative wall thickness by $k=a / b$, and, passing to normal stresses, we finally obtain:

$$
p_{g r}=\frac{2}{3} \sigma_{0}\left(1-k^{3}\right) \frac{2 a \lambda_{T, g}+9}{2 a \lambda_{T, g}+6} .
$$

In this case, from the boundary conditions, we have on the inner plane $\sigma_{r}^{g r}=-p_{g r}$, then from (2) and (3) it follows 


$$
\sigma_{\varphi}^{g r}=\sigma_{\theta}^{g r}=\frac{1}{3} \sigma_{0}\left(1+2 k^{3}\right) \frac{2 a \lambda_{T, g}+9}{2 a \lambda_{T, g}+6} .
$$

Using the classic condition von Mises $\left.T\right|_{r=a}=T_{0}$, what determines the corresponding pressure

$$
p_{0}=\frac{2}{3} \sigma_{0}\left(1-k^{3}\right)
$$

Moreover, on the inner plane: $\sigma_{r}=-p_{0}$, a

$$
\sigma_{\varphi}=\sigma_{\theta}=\frac{1}{3} \sigma_{0}\left(1+2 k^{3}\right)
$$

Comparing (5) and (6), we are convinced that the elastic work area of the material due to deformations constraint is increased regardless of the walls' relative thickness. At the same time, the increase in the limiting stress does not depend in any way on the wall thickness, which fundamentally distinguishes the reservoir from bent plates and beams [6-12], where the cross-section height dominantly determines the deformation restraint degree.

In addition to (1), to describe the constraint effect, other functions can be used for approximation, which, by definition, makes it possible to investigate the qualitative properties of the described object, focusing on numerical characteristics, reducing the problem to studying simpler or more convenient objects [16]. The only value of the increased voltage $\sigma_{g r}$ which in our proposed model should be within $0 \leq \sigma_{g r} \leq 1.5 \sigma_{0}$ is reliably known from the experimental data [2]. Within these limits, it is necessary to select for technical approximation algebraic dependences that are quite simple in the mathematical sense, containing no more than one approximation coefficient associated with experimental data.

In the most general form for the problem under consideration, this can be written as

$$
T_{g r}=T_{0}[1+\lambda f(r)]
$$

where $\lambda$ is the approximation coefficient, and $f(r)$ is some algebraic function of the radius. Then, in the generalized case (5) takes the form

$$
p_{g r}=\frac{2}{3} \sigma_{0}\left(1-k^{3}[1+\lambda f(r)]\right.
$$

and

$$
p_{g r} / p_{0}=1+\lambda f(r)
$$

In [17-18], some functions that, in relation to the problem under consideration, lead (9) to the following dependencies, are proposed:

- fractional linear 


$$
p_{g r} / p_{0}=\frac{20.1587 \cdot 2 a+9}{20.1587 \cdot 2 a+6}
$$

- irrational

$$
p_{g r} / p_{0}=1+\frac{0.1706}{\sqrt[4]{a}}
$$

- sedate

$$
p_{g r} / p_{0}=\frac{3 \sqrt[a]{1.093}-1}{2 \sqrt[a]{1.093}}
$$

- logarithmic

$$
p_{g r} / p_{0}=1+0.2 \lg \frac{0.9297 a+3}{0.9297 a}
$$

or

$$
p_{g r} / p_{0}=1+0.1 \ln \frac{1.5191 a+3}{1.5191 a}
$$

- trigonometric

$$
p_{g r} / p_{0}=1.5-0.5 \operatorname{tg} \frac{27.8565 a}{27.8565 a+3} \cdot \frac{\pi}{4}
$$

When using any of these dependencies, regardless of the wall thickness at $a \rightarrow 0 ; p_{g r} / p_{0}$ $\rightarrow 1.5$; at the large values $a p_{g r} / p_{0} \rightarrow 1$.

The numerical values for the excess according to (10) - (15) for the tanks with real values of the internal cavity are summarized in Table 1 . The lower rows of the table show the results of mathematical processing of the values array by analogy with the direct experimental data set. The arithmetic mean of the number of functions $n=6$ determines the absolute random error between the approximating functions. The specified hit reliability is $p=0,95$, Student's coefficient $t=2,4$. The final result in the last row, taking into account rounding, means that the required excess variation of the bearing capacity lies in the confidence interval with the probability $95 \%$.

Table 1. Increase values $p_{g r} / p_{0}$ for various approximations in (10)-(15)

\begin{tabular}{|l|c|c|c|c|c|c|}
\hline \multirow{2}{*}{$p_{g r} / p_{0}$} & \multicolumn{7}{|c|}{ Inner radius $a, \mathrm{~m}$} \\
\cline { 2 - 7 } & 5.0 & 1.0 & 0.5 & 0.25 & 0.1 & 0.01 \\
\hline by (10) & 1.015 & 1.065 & 1.115 & 1.187 & 1.299 & 1.469 \\
\hline by (11) & 1.114 & 1.171 & 1.203 & 1.241 & 1.303 & 1.5 \\
\hline
\end{tabular}




\begin{tabular}{|l|c|c|c|c|c|c|}
\hline by (12) & 1.009 & 1.043 & 1.081 & 1.15 & 1.295 & 1.499 \\
\hline by (13) & 1.043 & 1.125 & 1.174 & 1.299 & 1.304 & 1.5 \\
\hline by (14) & 1.033 & 1.109 & 1.16 & 1.218 & 1.303 & 1.5 \\
\hline by (15) & 1.016 & 1.071 & 1.123 & 1.194 & 1.301 & 1.467 \\
\hline average & 1.038 & 1.097 & 1.143 & 1.203 & 1.301 & 1.489 \\
\hline $\begin{array}{l}\text { absolute } \\
\text { random } \\
\text { error }\end{array}$ & 0.038 & 0.046 & 0.043 & 0.033 & 0.002 & 0.014 \\
\hline $\begin{array}{l}\text { final } \\
\text { result }\end{array}$ & $1.04 \pm 0.04$ & $1.1 \pm 0.05$ & $1.14 \pm 0.04$ & $1.2 \pm 0.03$ & $1.3 \pm 0$ & $1.49 \pm 0.01$ \\
\hline
\end{tabular}

The curves showing that at real dimensions $a=0.5 \div 5 \mathrm{~m}$ the excess is respectively $14 \div 4 \%$ are plotted in Fig. 2, based on the final results of the last row of Table 1 for the tanks made of the material with $\sigma_{0}=260 \mathrm{MPa}, \lambda_{T, g}=20.1587 \mathrm{~m}^{-1}$.

The numerical values of the material parameter (approximation coefficient) for (11) - (15), respectively, $\quad$ are $\quad \lambda_{u p}=0.1706\left[\mathrm{~m}^{1 / 4}\right] ; \quad \lambda_{c m}=1.093\left[\mathrm{~m}^{1 / a}\right] ; \lambda_{\log }=0.9297\left[\mathrm{~m}^{-1}\right]$; $\lambda_{\mathrm{ln}}=1.5191\left[\mathrm{~m}^{-1}\right] ; \lambda_{\mathrm{tg}}=27.8565\left[\mathrm{~m}^{-1}\right]$.

Under the influence of external pressure $q$ the tense state [14]

$$
\sigma_{r}=B \frac{r^{3}-a^{3}}{r^{3}} ; \sigma_{\varphi}=\sigma_{\theta}=B \frac{2 r^{3}+a^{3}}{2 r^{3}}
$$

gives

$$
T=\sqrt{3 B^{2}} a^{3} / 2 r^{3},
$$

where

$$
B=\frac{q b^{3}}{a^{3}-b^{3}},
$$

then

$$
\operatorname{grad} T=\left|\frac{\partial T}{\partial r}\right|=3 \sqrt{3 B^{2}} a^{3} / 2 r^{4},
$$

but, as in the previous case,

$$
\frac{\operatorname{grad} T}{T}=\frac{3}{r} .
$$

As $\left.T\right|_{r=a}>\left.T\right|_{r=b}$, fluidity occurs on the inner edge of the reservoir and substitution of the last expression in (1) again leads to the relation (4) with the same interpretation. The quantity $q_{g r}$ is expressed similarly and coincides with the right-hand side of (5), but at the same time on the surface of the cavity $\sigma_{r}^{g r}=0$, and from (16) it follows 


$$
\sigma_{\varphi}^{g r}=-\sigma_{0} \frac{2 a \lambda_{T, g}+9}{2 a \lambda_{T, g}+6}
$$

With the usual approach $\left.T\right|_{r=a}=T_{0}$, what determines $q_{0}$ similar to the expression (6) and gives $\sigma_{r}=0$, and $\sigma_{\varphi}=-\sigma_{0}$.

The latter results can be used to assess the effect of stress concentration near a spherical cavity in a ball of sufficiently large dimensions at $a \rightarrow 0$. In this case $k \rightarrow 0$ and the solution is not related to the size of the outer contour. Then from (16) we come to the wellknown solution $\sigma_{\varphi}=-1,5 q$. By virtue of this consideration and (5), it follows from (17) that the compressive circumferential stresses in both longitude and polar distance, in order to achieve the onset of plastic flow, in contrast to the classical solution, should exceed the value of the yield stress, namely: $\sigma_{\varphi}=\left.\sigma_{\theta}\right|_{r=a \rightarrow 0} \rightarrow-1,5 \sigma_{0}$.

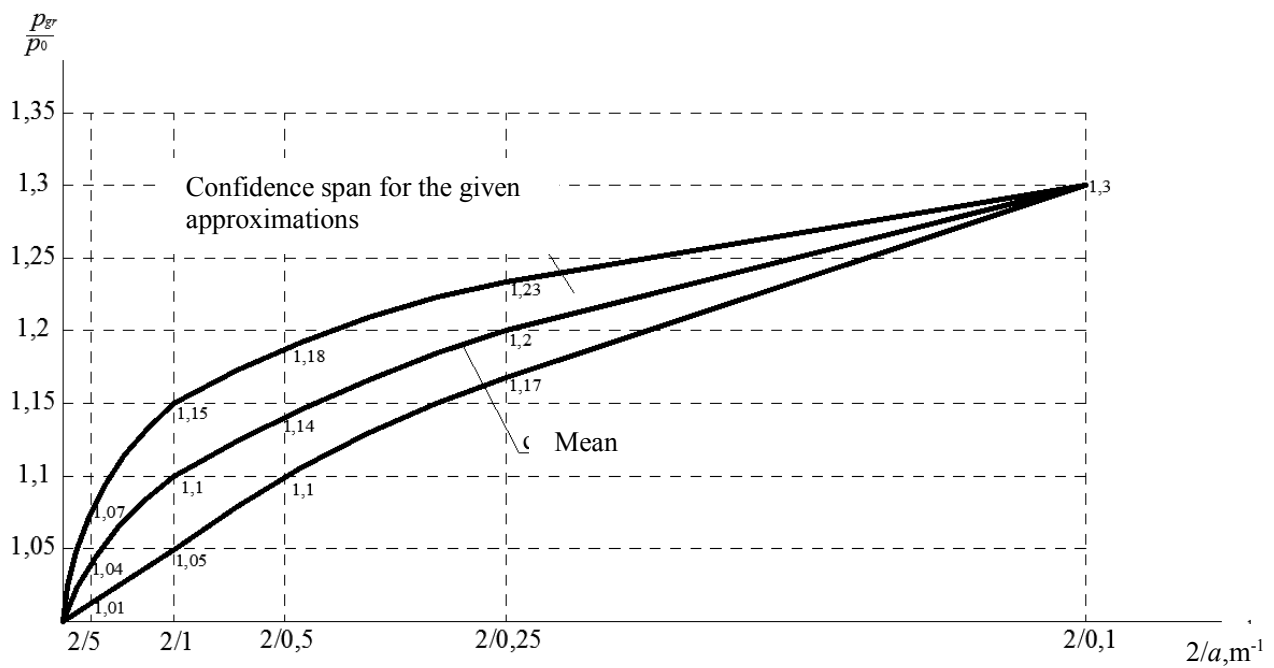

Fig. 2. The graph of the increase in the bearing capacity of a spherical tank depending on the inner cavity radius

The work was carried out by order of the Federal State Budgetary Institution "TsNIIP of the Ministry of Construction of Russia" on the topic 7.41.21 "Plan of fundamental scientific research of RAASN and the Ministry of Construction and Housing and Communal Services of the Russian Federation" for 2020.

\section{References}

1. V.A. Baldin, Structural mechanics and calculation of structures 1, 29-31 (1977)

2. V.A. Baldin, V.N. Potapov, A.A. Fadeev, Structural mechanics and calculation of structures 1, 29-31 (1977) 
3. A.A. Fadeev, On the transition of low-carbon steel to an elastic-plastic state with an uneven distribution of stresses (with pure bending) (Investigation of the strength of elements of building metal structures: Proceedings of the Institute, Central Research Institute of Building Structures named after V.A. Kucherenko. M., 1982)

4. F. Campus, Bull. of the Sciences class of the R. de Belgique Academy 5, 49 (4), 303-314 (1963)

5. N.M. Dehousse, Bull. of the Sciences class of the R. de Belgique Academy 5 (48), 329334 (1962)

6. G.A. Geniyev, S.Yu. Kalashnikov, The influence of stress gradients, geometry and crosssection scales on the transition of bending elements into a plastic state, Research in structural mechanics: Proceedings of the Institute, (Central Research Institute of Building Structures named after V.A. Kucherenko M., 1985)

7. G.A. Geniyev, S.Yu. Kalashnikov, Structural mechanics and calculation of structures 6, 12-15 (1988)

8. G.A. Geniyev, S.Yu. Kalashnikov, On the construction of incremental plasticity conditions, Central Research Institute of Building Structures named after V.A. Kucherenko, M., 1984)

9. S.Yu. Kalashnikov, Proceedings of NSACU, Novosibirsk 6 (6) (27), 142-148 (2003)

10. S.Yu. Kalashnikov, A.V. Levin, On the solution of problems of plane transverse bending using the incremental plasticity condition, (Volgograd State University of Architecture and Civil Engineering, Volgograd, 1988)

11. S.Yu. Kalashnikov, On the solution of some two-dimensional problems using the incremental plasticity condition (Central Research Institute of Building Structures named after V.A. Kucherenko M., 1984)

12. S.Yu. Kalashnikov, E.V. Gurova, S.A. Kalinovskiy, Bulletin of the Volgograd State University of Architecture and Civil Engineering, Series: Building and architecture 4 (81), 4-12 (2020)

13. S.Yu. Kalashnikov, News of Higher Educational Institutions, North Caucasian region. Technical science 44-48 (2003)

14. S.P. Timoshenko, J. N. Goodier, Theory of elasticity (Translated from English by M.I. Reitman. M., Science, 1979).

15. G. Lame, Lessons on the Theory of Elasticity (Paris, 1852)

16. A.N. Golubinskiy, Bulletin of the Voronezh Institute of the Ministry of Internal Affairs of Russia 2, 138-143 (2007)

17. S.Yu. Kalashnikov, V.P. Vatanskiy, Alternative approximating functions of the incremental theory of nonlinear deformation of bodies, Materials of the annual scientificpractical conference of the faculty and students of VolgSACU, April 24-27, 2007: at $3 \mathrm{pm}$ Part 2: Natural sciences. Construction production technology. Heat, gas and water supply, VolgSACU, Volgograd, 2008. 270-272 (2020)

18. S.Yu. Kalashnikov, Experimental verification of the material deformation model under conditions of an inhomogeneous stress state: monograph (VolgSTU, Volgograd, 2017) 